\section{Counterpoint: highly-sensitive troponin immunoassays in the emergency department}

\section{Giuseppe Lippi, ${ }^{1}$ Gianfranco Cervellin ${ }^{2}$}

${ }^{1}$ Laboratory of Clinical Chemistry and Hematology, Pathology and Laboratory Medicine Department, Parma University Hospital; ${ }^{2}$ Emergency Department, Parma University Hospital, Parma, Italy

\section{Dear Editor,}

The introduction of the cardiospecific troponin(s) testing at the beginning of the $21^{\text {st }}$ century has patently revolutionized the diagnostic approach to acute coronary syndrome (ACS), and has now become the cornerstone for diagnosing acute myocardial infarction (AMI), especially in patients with atypical symptoms, non-diagnostic electrocardiogram (ECG) and/or non-ST elevation myocardial infarction (NSTEMI). ${ }^{1}$ An unremitting refinement of the analytical characteristics of earlier troponin immunoassays has finally led to development and commercialization of the socalled latest generation or highly-sensitive (HS) methods. The former term should be used to define those methods that allow the measurement of $99^{\text {th }}$ percentile of the protein in the reference population with an imprecision $\leq 10 \%$, whereas the latter definition refers to those commercial tests that allow the measurement of the protein in $\geq 75 \%$ of apparently healthy adults representing the normal reference population. ${ }^{2}$ To rephrase this, it could be said that the test was black or white until a few years ago, as it was only capable to distinguish between AMI and non-AMI patients. At present, instead, we should learn to deal with a grey scale test, since cardiac troponin(s) levels reflect a continuum of myocardial damage, rather than being a simple dichotomous biomarker (Figure 1). Although some unquestionable advantages have emerged from the use of HS troponins, yet there are some drawbacks that are now putting to the test several emergency physicians, as well as some laboratory professionals.

The major problem became apparent after the introduction of HS troponin immunoassays for diagnosing AMI in the emergency department (ED). The problem is represented by the lack of diagnostic specificity for identifying an ischemic injury. Protein(s) concentrations evidently increase in a variety of non-ischemic cardiac disorders - including cardiac contusions, myocarditis, rhabdomyolysis, severe arrhythmias, valvular diseases, aortic dissection, cardiomiopathies, infiltrative disorders and cardiotoxicity by drugs, poisons or chemotherapy. ${ }^{3,4}$ Besides that, there is a kalei- doscope of extra-cardiac conditions, either physiological or pathological, which may justify the release of cardiac troponin(s) to such an extent that may become actually measurable with most HS immunoassays, and which should be clearly identified to prevent overcrowding in the EDs. With respect to the physiological variables, several studies have clearly shown that healthy ageing and gender differences are associated with different values of cardiac troponin(s). In an article published 4 years ago, Giannoni et al. already emphasized that the concentration of troponin(s) steadily increases across decades of ages, and is consistently higher in males than females, with values being nearly 15 -fold higher in healthy men over 70 as compared with healthy women under $20 .^{5}$ More recently, increased values of HS troponin in older men were also reported by Normann et al., as a result of a higher prevalence of silent cardiovascular disease in this population, an aspect that might dramatically unbalance the diagnostic performance of these biomarkers, especially for identifying NSETMI. ${ }^{6}$ According to this data, it seems reasonable to put forward the hypothesis that further practical refinements may be necessary to enhance the diagnostic performance of $\mathrm{HS}$ immunoassays in ED patients. Incidentally, in most cases the upper reference limit (URL) of the novel HS method has been calculated by the diagnostic industry - and subsequently in preclinical validation studies settings - using a presumably healthy population, which does not correspond to the real world (i.e. the ED), where these tests are mostly used. In a recent article, we have shown that $99^{\text {th }}$ percentile of the URL recalculated on a population of ED patients with no signs or symptoms of myocardial ischemia was nearly 4 -fold higher (i.e. 32.7 vs $8.6 \mathrm{ng} / \mathrm{L}$ ) than that originally proposed and validated for the same assay. ${ }^{7}$ As such, partitioning of diagnostic thresholds according to age and gender appears a virtually unavoidable step to facilitate the meaningful use of HS troponin immunoassays in the foreseeable future. Meticulous troubleshooting of nonischemic and/or extra-cardiac causes of increase is another necessary action, which is something that we were not used to with the former contemporary-sensitive methods. This obviously requires appropriate and continuous education of emergency physicians about the known causes of troponin increase, considering that the adoption of disease-specific thresholds seems unpractical due to the vast number of conditions that may enhance a physiological or pathological turnover of cardiomyocytes or decrease protein catabolism such as renal and liver failure, ${ }^{8}$ hypertension, hypothyroidism, ${ }^{1}$ traumatic brain injury, ${ }^{9}$ along with physical activity. ${ }^{10}$

Another important question is the improper use of the current nomenclature. Despite the
Correspondence: Giuseppe Lippi, Laboratory of Clinical Chemistry and Hematology, Pathology and Laboratory Medicine Department, Parma University Hospital, via Gramsci 14, 43126 Parma, Italy.

Tel. +39.0521 .703050 - Fax: +39.0521 .703791 .

E-mail: glippi@ao.pr.it, ulippi@tin.it

Key words: myocardial infarction, troponin, immunoassays, high-sensitivity.

Contributions: the authors contributed equally.

Conflict of interests: the authors declare no potential conflict of interests.

Note: this Letter to the Editor relates to Plebani M, Zaninotto M. Point: high-sensitive troponin assays - a valuable tool for astute physicians. Emerg Care J 2013;9:e15

Received for publication: 30 April 2013. Accepted for publication: 27 May 2013.

This work is licensed under a Creative Commons Attribution 3.0 License (by-nc 3.0).

(C) Copyright G. Lippi and G. Cervellin, 2013

Licensee PAGEPress, Italy

Emergency Care Journal 2013; 9:e16

doi:10.4081/ecj.2013.e16

claims issued by scientific societies aming at harmonizing the terms according to genuine analytical evidence, a variety of improbable and often bizarre terms are being used to designate the analytical sensitivity of some commercial methods by the in vitro diagnostic industry, including the use of weird prefixes such as ultra-, modified-, extra- ${ }^{11}$ It is rather clear that all these terms may cause a comprehensible confusion in the already crowded minds of emergency physicians, who have little time - and even less interest - to spend for understating whether an ultra-sensitive assay is more efficient than an extra-sensitive method or vice versa. What should be simply clear to everybody - including manufacturers, laboratory professionals, cardiologists and emergency physicians - is which cut-off value should be used for diagnosing AMI in ED patients, regardless of the claimed sensitivity of the method. It is almost irrelevant that the limit of detection of the method is $20 \mathrm{ng} / \mathrm{mL}$ or 10 times lower (i.e. $2 \mathrm{ng} / \mathrm{L}$ ), if the diagnostic threshold is ultimately comparable. A paradigmatic example has already been provided, where the optimal cut-off for diagnosing AMI in the ED and the $99^{\text {th }}$ percentile URL in males of a contemporary-sensitive method (limit of detection $10 \mathrm{ng} / \mathrm{L}$, measurable values attainable in $35 \%$ of a healthy population) were proven to be paradoxically lower than those of the latest development of the same immunoas- 
say to fulfill HS-characteristics (limit of detection $2.5 \mathrm{ng} / \mathrm{L}$, measurable values attainable in $80 \%$ of a healthy population). ${ }^{11,12}$ Another good example of the gap existing between what is theorized and what is being daily practiced comes from a recent and interesting study by Venge and Lindahl, who showed that a commercial assay defined as not (clinically) acceptable displayed equivalent or even superior ability for identifying patients with poor outcomes as compared with other commercial methods that are currently ranked as guideline acceptable from a genuine analytical perspective. $^{13}$

Using HS methods, the emergency physicians have to deal with a new diagnostic dilemma: how can we separate patients with AMI and initially elevated cardiac troponin(s) level from those who have levels above the cut-off, resulting from non-ACS causes? Probably, the best answer can be found in an appropriate serial testing, although the appropriate definition of timing in serial testing and protein increase is indeed another matter of debate (and confusion), using HS troponins(s). ${ }^{14}$ Considering that the protein concentration has now become measurable in the vast majority of subjects regardless of being healthy or diseased - and that several ED patients suffering from nonischemic disorders display values largely exceeding the $99^{\text {th }}$ percentile URL when HS methods are used, the use of serial sampling for establishing troponin kinetics is vital. Several studies have proposed rather different approaches, including heterogeneous sampling times (with 1, 2, 3, 4 and even $6 \mathrm{~h}$ intervals), combined with broad reference change values, from $20 \%$ (as for the former recommendations of the US National Association of Clinical Biochemistry) to over $150 \% .{ }^{4}$ Absolute rather than percentage increase of values has also been proposed. This plethora of numbers and approaches is not informative. In fact, it plunges the most appropriate use and setting of HS immunoassays into confusion. It is also noteworthy that the firm paradigm that HS methods would enable a faster rule out of ACS in ED patients has been recently questioned. With respect to this, Cullen et al. showed that an algorithm incorporating troponin concentration and delta values with a contemporary-sensitive method allowed accurate diagnosis of AMI and a faster exclusion of ischemic heart disease in the majority of ED patients within $2 \mathrm{~h}$ from presentation. $^{15}$

All this considered, it is not so naive to cast some doubts on the introduction of a HS method as always increasing the number of early diagnoses of AMI in the ED, at least upon patient admission. This may obviously depend on the real gap existing between the clinical and analytical characteristics of the previous assay and those of the latest (Figure 1). As the contemporary-sensitive assay already provides

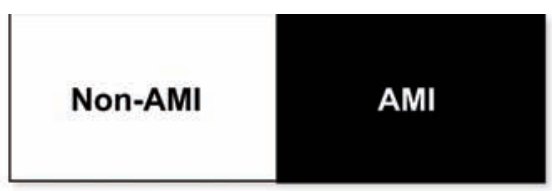

First generation troponin immunoassays

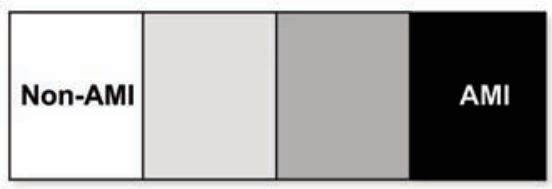

Latest generation troponin immunoassays
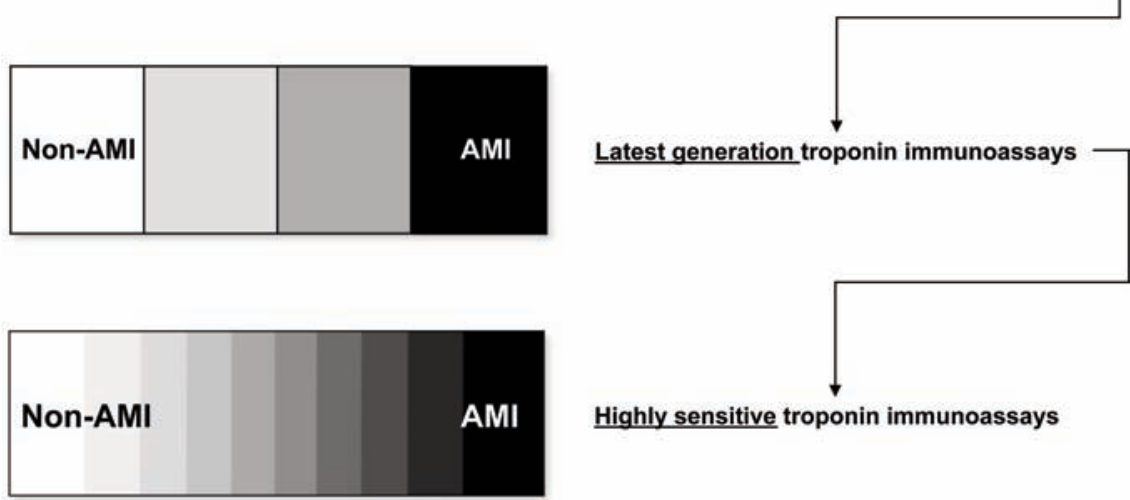

Highly sensitive troponin immunoassays

Figure 1. Evolution of troponin immunoassays for diagnosing acute myocardial infarction.

good clinical performance, the reasons in support of a transition toward an HS method are probably flawed. It is in fact undeniable that what can be optimistically gained in terms of sensitivity, will then be lost in terms of specificity, because a larger number of measurable values that are attainable in patients with nonischemic disorders would negatively impact on the effective rate of rule-out.

An important aspect to be considered by those healthcare professionals working outside the laboratory environment - and that is expected to expand exponentially in the near future - is the limited possibility of choosing between one troponin immunoassay and another in a world of limited resources. Tenders and other forms of acquisition of laboratory reagents and instrumentation are now spreading around the concept of core laboratories, where troponin testing will remain an important player, but where it also may be sacrificed in favor of healthcare economy and laboratory organization. ${ }^{16}$

It is really suggestive - or even bizarre according to a subjective perspective - that the development of the latest generation troponin immunoassays have been propelled by three driving forces, i.e. the cardiologists' aspiration to increase the number of effective diagnosis of myocardial ischemia, the laboratorists' inclination to use the best possible analytical method, and the competition among different manufacturers to fulfill these needs. Still, has anyone ever asked what emergency physicians do really think and need for their daily struggle in overcrowded emergency rooms?

\section{References}

1. Lippi G, Franchini M, Cervellin G. Diagnosis and management of ischemic heart disease. Semin Thromb Hemost 2013;39:202-13.

2. Casagranda I, Cavazza M, Clerico A, et al. Latest generation cardiac troponins in patients with suspected acute coronary syndrome without persistent ST-segment elevation: proposal of use in emergency departments. Emerg Care J 2013;9:39-45.

3. Lippi G, Cervellin G. Identification of troponin determinants for improving its diagnostic performance in the emergency department. J Emerg Med 2012;43:e487-8.

4. Lippi G, Montagnana M, Aloe R, Cervellin G. Highly sensitive troponin immunoassays: navigating between the scylla and charybdis. Adv Clin Chem 2012;58:1-29.

5. Giannoni A, Giovannini S, Clerico A. Measurement of circulating concentrations of cardiac troponin I and $\mathrm{T}$ in healthy subjects: a tool for monitoring myocardial tissue renewal? Clin Chem Lab Med 2009; 47:1167-77.

6. Normann J, Mueller M, Biener M, et al. Effect of older age on diagnostic and prognostic performance of high-sensitivity troponin $\mathrm{T}$ in patients presenting to an emergency department. Am Heart J 2012;164: 698-705.

7. Lippi G, Margapoti R, Aloe R, Cervellin G. Highly-sensitive troponin I in patients admitted to the emergency room with acute infections. Eur J Intern Med 2013;24: e57-8. 
8. Lippi G, Cervellin G, Plebani M. Sensitive cardiac troponin T assay. New Engl J Med 2010;362:1242.

9. Lippi G, Dipalo M, Carbucicchio A, et al. The concentration of highly-sensitive troponin I is increased in patients with brain injury after mild head trauma. Int J Cardiol 2013;168:1617-8.

10. Lippi G, Cervellin G, Banfi G, Plebani M. Cardiac troponins and physical exercise. It's time to make a point. Biochem Med (Zagreb) 2011;21:55-62.

11. Apple FS, Ler R, Murakami MM. Determination of 19 cardiac troponin I and
T assay 99th percentile values from a common presumably healthy population. Clin Chem 2012;58:1574-81.

12. Lippi G, Cervellin G, Robuschi E, et al. Comparison of high sensitivity and contemporary troponin I immunoassays for the early detection of acute myocardial infarction in the emergency department. Ann Clin Biochem 2012;49:205-6.

13. Venge P, Lindahl B. Cardiac troponin assay classification by both clinical and analytical performance characteristics: a study on outcome prediction. Clin Chem 2013;59: 976-81.
14. Lippi G, Cervellin G. Letter by Lippi and Cervellin regarding article, high-sensitivity cardiac troponin in the distinction of acute myocardial infarction from acute cardiac noncoronary artery disease. Circulation 2013;127:e353.

15. Cullen L, Parsonage WA, Greenslade J, et al. Delta troponin for the early diagnosis of AMI in emergency patients with chest pain. Int J Cardiol (2013) (in press).

16. Lippi G, Cervellin G. Letter to the Editor: choosing troponin immunoassays in a world of limited resources. J Am Coll Cardiol 2013;62:647-8. 